\title{
Efficacy of Transcranial Direct Current Stimulation Coupled with a Multidisciplinary Rehabilitation Program for the Treatment of Fibromyalgia
}

\author{
Marcelo Riberto ${ }^{*}$, , Fabio Marcon Alfieri ${ }^{2}$, Kátia Monteiro de Benedetto Pacheco ${ }^{1}$, Valeria Dini Leite ${ }^{1}$, \\ Harumi Nemoto Kaihami ${ }^{1}$, Felipe Fregni ${ }^{3}$ and Linamara Rizzo Battistella ${ }^{1}$ \\ ${ }^{I}$ Instituto de Medicina Física e Reabilitação - Universidade de São Paulo, Brazil \\ ${ }^{2}$ Universidade Adventista de São Paulo, Brazil \\ ${ }^{3}$ Harvard University, Boston, MA, USA
}

\begin{abstract}
Pain control in fibromyalgia patients is limited no matter the therapeutic regimens used. Recent data have shown that daily sessions of anodal transcranial direct current stimulation (tDCS) over the primary motor cortex (M1) in patients with fibromyalgia (FM) are associated with reduction of pain perception.

Objective: We aimed to test whether active tDCS, as compared with sham tDCS, combined with multidisciplinary rehabilitation is associated with significant clinical gains in fibromyalgia.

Design: This was a randomized, double-blinded controlled trial.

Subjects: 23 patients were randomized to receive weekly sessions of multidisciplinary rehabilitation approach combined with sham or anodal tDCS of M1. Patients were evaluated for pain with VAS and for quality of life with SF-36, fibromyalgia pain questionnaire and health assessment questionnaire by a blinded rater before and after the 4 month period of rehabilitation.

Results: Patients tolerated tDCS treatment well, without adverse effects. Patients who received active treatment had a significantly greater reduction of SF-36 pain domain scores $\left(\mathrm{F}_{(2,21)}=6.57 ; \mathrm{p}=0.006\right)$ and a tendency of higher improvement in Fibromyalgia Impact Questionnaire (FIQ) scores after $(\mathrm{p}=0.056)$ as compared with sham tDCS/standard treatment, but no differences were observed in the other domains.

Conclusions: Although active tDCS was associated with superior results in one domain (SF-36 pain domain), the lack of significance in the other domains does not fully support this strategy (weekly tDCS) combined with a multidisciplinary approach.
\end{abstract}

Keywords: Electrical stimulation, cerebral cortex, fibromyalgia, pain, quality of life, rehabilitation.

\section{INTRODUCTION}

Fibromyalgia is characterized by chronic widespread pain, mainly on musculoskeletal structures, and multiple additional clinical manifestations [1]. The most accepted pathophysiologic model considers imbalance between nociception and normal physiologic pain control [2]. According to this model, there is an overall decrease in the inhibitory pain-related pathways; allowing, thus, low intensity or non-nociceptive stimuli to be processed in precortical and cortical pain-related structures involved in the affective and cognitive processing of pain [3]. The net result is an increase in pain perception. Based on this theory, therapeutic approaches aiming at the modulation of central nervous system might be beneficial. In fact the development of techniques of non-invasive brain stimulation has provided

*Address correspondence to this author at the Department of Biomechanics, Medicine and Rehabilitation of the Locomotor System, School of Medicine in Ribeirão Preto, University of São Paulo, Avenida Bandeirantes 3900, Monte Alegre CEP14048-900, Ribeirão Preto, SP, Brazil; Tel: (5516) 36022416; E-mail: mriberto@usp.br a means for such modulation. One technique that has been studied for the treatment of chronic pain is transcranial direct current stimulation (tDCS) [4-12].

In transcranial direct current stimulation (tDCS), a pair of electrodes is placed on the scalp and low intensity continuous current flows between them [4]. Depending on the electrodes location, it has been shown that this technique induces significant currents in cortical areas that induce significant changes in cortical excitability [6]. Several human studies have shown that tDCS induces changes in cortical excitability depending on the current orientation; such as that anodal stimulation increases cortical excitability and cathodal stimulation decreases it $[5,13]$. These excitability changes can be explained by synaptic changes and direct effects on neuronal spontaneous activity [7].

Based on the noninvasive and safe profile of this technique; several studies have been conducted testing the efficacy of tDCS for the treatment of chronic pain including pain in spinal cord injury [8] and fibromyalgia $[9,14]$. In fact, in a previous study, Fregni et al. [9] showed that 20 minutes of daily direct anodal stimulation over the primary 
motor cortex for 5 days is effective to decrease pain in patients with fibromyalgia as compared with sham and to active prefrontal tDCS. Because the mechanisms of action of tDCS that induces a change in neuronal spontaneous activity; the use of this technique coupled with behavioral interventions might enhance its effect as demonstrated before in motor learning [10], speech therapy [11] and working memory [15]. Therefore we hypothesized that fibromyalgic subjects under in a rehabilitation program for pain would achieve greater benefit as indexed by pain and quality of life scales if they were submitted to anodal tDCS of M1 rather than sham stimulation, active tDCS of M1 coupled with a rehabilitation program for pain would result in a greater benefit as indexed by pain and quality of life scales as compared with sham tDCS coupled with the same rehabilitation program.

\section{MATERIALS AND METHODOLOGY}

\section{Study Design and Participants}

We performed a randomized, double blind, single-center placebo controlled trial. This study has been approved by the local ethics committee, in accordance with the Helsinki declaration, and all the subjects signed a written informed consent form.

Our inclusion criteria were: 1) females who fulfilled the American College of Rheumatology criteria for fibromyalgia, because fibromyalgia is much more prevalent in women, the restrict male participation was based on convenience; 2) Aged from 18 to 65 years old; 3) Able to participate in the rehabilitation program for fibromyalgia. We excluded patients with psychiatric and behavioral conditions severe enough to limit the participation in group activities and those with other restrictive health conditions. Finally patients with cardiovascular limitation for exercise were also excluded, because treatment is mostly based on cardiovascular training.

Sample size calculation was based on the assumption of significance of 0.05 and power of 0.80 . According the Fregni results [9], a mean reduction of 3 points in VAS for the group under active stimulation was expected, in contrast to no improvement in the sham group. This resulted in 2 groups with 10 participants. We decided to add 2 more patients in each group in order to prevent any reduction of power in case of patient desistence. Finally, we enrolled 23 patients to participate in the study (Table 1 describes demographic characteristics). They underwent the same multidisciplinary rehabilitation program for pain and were randomly assigned to active $\mathrm{tDCS}(\mathrm{A})$ or sham $(\mathrm{S})$ using a simple randomization method.

\section{Rehabilitation Program for Pain}

All subjects participated in the same rehabilitation program for pain, which has lasts 4 months and is comprised by the following routine: during the first hour subjects have educative interventions or cognitive behavior group therapy focused in pain. Subjects are oriented on posture and ergonomics by physical and occupational therapists and in behavioral modulation by psychologists and social workers, and during the second hour they participate in cardiovascular and strengthening training or stretching exercises. Subjects have these sessions 3 times a week. This program is the standard treatment in our clinic at University of Sao Paulo as has shown before to be effective [16].

\section{Transcranial Direct Current Stimulation}

Direct current was transferred by a saline-soaked pair of surface sponge electrodes $\left(35 \mathrm{~cm}^{2}\right)$ and delivered by a specially developed, battery-driven, constant current stimulator. Patients were randomized to two different types of treatment:

1) Anodal stimulation of primary motor cortex (M1) the anode electrode was placed over C3 (EEG 10/20 system) and the cathode electrode over the contralateral supraorbital area - similar to the montage of the study of Fregni et al. [8,14].

2) Sham stimulation of M1 - For sham stimulation, the electrodes were placed in the same positions as for anodal M1 stimulation; however, the stimulator was turned off after 30 seconds of stimulation. Therefore the subjects felt the initial itching sensation, but received no current for the rest of the stimulation period. A recent study showed that this method of sham stimulation is reliable [17].

Because we assumed that tDCS may act as a facilitator of behavioral modifications by establishing a favorable neural environment, all the stimulations were performed before the first hour of activities of the rehabilitation program. A constant current of $2 \mathrm{~mA}$ intensity was applied for 20 minutes once a week for 10 weeks. The rationale for weekly frequency was the intention to couple stimulation with the activities of the multidisciplinary program and because daily stimulation would not be feasible. The device used for electric stimulation had a knob to allow the operator to control current intensity. Stimulation was ramped up or down in no more than 5 seconds. Stimulation with $2 \mathrm{~mA}$ (for a single session) has been shown to be safe in healthy volunteers [18].

\section{Assessments}

A blinded evaluator used the following instruments to score pain and quality of life in these patients immediately before and after the 4 month therapeutic program:

Visual analogue scale for pain (VAS) consists of a 100 $\mathrm{mm}$ line with the expressions "no pain" and "worst pain" in each extremity. Subjects were requested to indicate the intensity of pain at the moment and that could be quantified in millimeters from the "no pain" extremity.

A dynamometer was used to determine the pressure threshold to elicit pain on the 18 fibromyalgia tender point a mean value was recorded as "tenderness index", which represented the mean pressure threshold to elicit pain [18].

Brazilian versions of quality of life (QoL) questionnaires:

- $\quad$ Fibromyalgia Impact Questionnaire (FIQ) - which is a multidimensional instrument that measures functional capacity for daily living and work, as well as the sensation of well-being, pain, anxiety, depression, morning stiffness and sleep quality. Its final score varies from 0 (lowest impact or less deficit to functional capacity) to 100 (highest impact) [19]. 
- SF-36 is a widely used QoL instrument which represents 8 generic health concepts: physical functioning, role-physical; bodily pain, general health, vitality, social functioning, role-emotional, and mental health - each of them is score from 0 (worst) to 100 (best) [20].

- Health Assessment Questionnaire (HAQ) disability index is directed to assess fine movements of the upper extremity, locomotor activities of the lower extremity, and activities that involve both the upper and lower extremities. Twenty questions on activities are distributed in 8 subscales, which are score in a 4 level graduation. The summary score varies from 0 (without any difficulty) to 3 (unable to do) [21].

- $\quad$ Beck depression inventory (BDI) and Hamilton rating scale for depression (HAM) were used to evaluate severity of depression as mood changes is an important confounder [22].

\section{Statistical Analysis}

Having tested homogeneity of variances of the main outcomes, we performed a mixed ANOVA model in which we considered the subject as a random effect; condition of treatment and time of treatment as a fixed effect. We therefore included the following fixed effect variables in the model: time (pre and post-stimulation); condition of stimulation (tDCS/standard treatment $v s$ sham tDCS/ standard treatment) and the interaction time*condition.

Unless stated otherwise, all results are presented as mean and standard deviation, and statistical significance refers to a two-tailed $\mathrm{p}$ value $<0.05$.

\section{RESULTS}

General characteristics of groups active and sham are shown in Table 1. Twelve patients were allocated to placebo stimulation and 11 to active stimulation, there were no patients lost to follow-up. It can be stated that subjects who received the active stimulation were older, had studied longer, their pain complaints lasted longer and were not economically active. Tricyclic antidepressants, selective serotonin reuptake inhibitors, muscle relaxants and paracetamol were equally used in both groups and there was no change in the use of such drugs with the treatment.

Table 1. General and Clinical Characteristic of Studied Subjects (Mean \pm SD)

\begin{tabular}{|c|c|c|}
\hline & Active & Sham \\
\hline \hline $\mathrm{N}$ & 11 & 12 \\
\hline Age (years) & $58.3 \pm 12.1$ & $52.4 \pm 11.5^{*}$ \\
\hline Formal education (years) & $6.3 \pm 5.2$ & $8.9 \pm 4.4^{*}$ \\
\hline Economically actives (\%) & 27.3 & $50^{*}$ \\
\hline Duration of widespread pain (months) & $9.9 \pm 11.8$ & $6.4 \pm 10.2^{*}$ \\
\hline $\mathrm{p}<0,05$. & & \\
\hline
\end{tabular}

\section{Pain Analysis}

For pain, we analyzed two outcomes - current pain levels as assessed by VAS and the pain domain of SF-36. The advantage of using SF-36 is that pain in this scale is measured longitudinally, because it takes into account the last 4 weeks. Indeed, mixed ANOVA model for these two dependent variables showed no significant interaction effect for VAS $(F(2,21)=0.4 ; p=0.67)$. Also, there was no statistically significant improvement in pressure tenderness until the end of the experiment in both groups, no interaction effect for dolorimetry was noticed $(\mathrm{F}(2,21)=0.8 ; \mathrm{p}=0.54)$. However, for pain measured by SF-36, there was a significant interaction effect $(\mathrm{F}(2,21)=6.57 ; \mathrm{p}=0.006)$. Posthoc tests showed that there was a significant reduction in pain after active tDCS/standard treatment $(p=0.006)$; but not after sham tDCS/standard treatment $(p=0.15)$ (see Fig. 1). Statistical significance could not be observed in the comparison of baseline mean values of VAS, SF-36 pain domain and pressure dolorimetry.

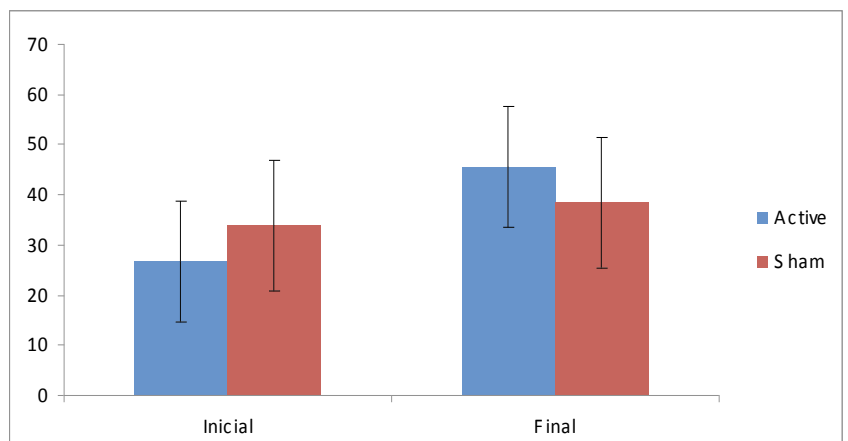

Fig. (1). SF-36 Pain domain scores (mean $\pm \mathrm{sd}$ ) before and after the intervention.

\section{Quality of Life}

The secondary outcome of this study was quality of life and fatigue. For the remaining scores of SF-36 (all except for pain), the interaction term was not significant $(\mathrm{F}(2,21)=0.03$; $\mathrm{p}=0.97$ ); however for FIQ, there was a trend for a significant interaction term time by group $(\mathrm{F}(2,21)=2.43 ; \mathrm{p}=0.11)$. We in an exploratory way, given that the interaction term was not significant, performed post-hoc comparisons for FIQ. Indeed there was a tendency for a significant decrease in FIQ scores after active tDCS/standard treatment $(\mathrm{p}=0.056)$, but not sham tDCS/standard treatment ( $p=0.18$ ) (Fig. 2).

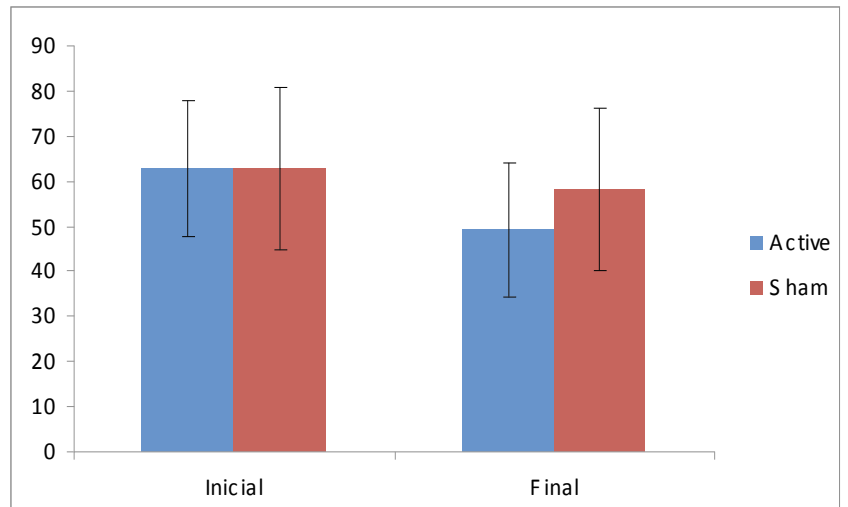

Fig. (2). FIQ scores (mean $\pm \mathrm{sd}$ ) before and after the intervention.

\section{Mood and Physical Disability}

We finally performed the same analyses; but used mood scales (HDRS and BDI) and the disability assessment (as 
indexed by HAQ) as the main outcomes. Although both groups showed marked significant improvement in physical capacity and mood (Table 2), further analyses showed a nonsignificant interaction term $(\mathrm{F}(2,21)=1.42 ; \mathrm{p}=0.31$ for HDRS; $\mathrm{F}(2,21)=2.05 ; \mathrm{p}=0.24$ for BDI and $\mathrm{F}(2,21)=1.53$; $\mathrm{p}=0.48$ for HAQ); suggesting that active tDCS did not induce any additional beneficial effect on these domains compared with sham tDCS and multidisciplinary rehabilitation program.

Table 2. Means Scores (Mean \pm SD) of HDRS, DBI and HAQ.

\begin{tabular}{|c|c|c|c|}
\hline & Inicial & Final & $\mathbf{p}$ \\
\hline \hline BDI & $18.1 \pm 11.2$ & $14.0 \pm 9.8$ & 0.01 \\
\hline HDRS & $20.2 \pm 8.5$ & $16.1 \pm 9.5$ & 0.04 \\
\hline HAQ & $1.52 \pm 0.47$ & $1.26 \pm 0.43$ & 0.0005 \\
\hline
\end{tabular}

\section{Correlations}

For the two dependent variables that showed some association with the type of treatment - pain/SF-36 and FIQ; we performed correlations between scores changes in the active group vs demographic and clinical characteristics (baseline pain and fibromyalgia pressure points). There were no significant correlations; however moderate correlations between pain/SF-36 and age $(\mathrm{r}=0.53, \mathrm{p}=0.09)$ and FIQ $v s$ educational level $(\mathrm{r}=-0.52, \mathrm{p}=0.097)$ were observed; such as that younger and more educated patients had a larger improvement.

\section{Blinding Evaluation}

At the end of each tDCS session, patients had to answer if they think they received active or sham stimulation. The subjects in both groups guessed at chance level (sham stimulation: only 5/11 could guess correctly and active stimulation: only $5 / 12$ could guess correctly). There were no significant differences across the two groups $(\mathrm{p}=0.72$, Fisher's exact test)

\section{DISCUSSION}

Because of its multiple clinical manifestations, which range from musculoskeletal pain to complaints on other body systems and psychological disorders, multidisciplinary intervention has been recommended as the best approach for patients with fibromyalgia [23]. The rationale for this intervention is that a sum of small gains can result in a significant improvement in the global functioning of these patients. Among the most recommended activities is cardiovascular training [24], educative effort to support the understanding of the disease and pain controls strategies [25] and pharmacological interventions with tricyclic antidepressants, selective serotonin reuptake inhibitors [26] or weak opiates [27]. The efficacy of multidisciplinary intervention to fibromyalgia has been demonstrated by different authors [23]. Due to the interference of pain in multiple aspects of functioning in these patients, composite instruments which involve dimensions such as pain, sleep quality, functional capacity and emotional status are recommended to evaluate the results of this intervention [28]. Parameters like HAQ, HDRS and BDI clearly improved, and FIQ showed a trend in the same direction, supporting the idea of global improvement in functional capacity, emotional aspects and reduction of the impact of fibromyalgia on functioning of these patients regardless the use of tDCS. These results on HAQ, HDRS and BDI reflect the beneficial effect of the multidisciplinary program both on psychological aspects and physical capacity, which result in an overall improvement of functioning.

Non-invasive brain stimulation has been proved to be effective for the reduction of pain in these patients in previous studies [9], so we tested whether noninvasive brain stimulation with tDCS could enhance the effects of the multidisciplinary program in pain reduction. The rationale for using tDCS to enhance this multidisciplinary program is based on the mechanisms of these two interventions [29]. The learning of new skills (in this case, reducing pain behaviors) is linked to changes in neuronal activity and excitability. They might reflect changes in synaptic strength, associated with long-term potentiation (LTP). Successful manipulation of cortical excitability to improve learning processes has been demonstrated in humans with tDCS [11]. TDCS presents an interesting alternative to these approaches, because it is non-invasive, painless and safe (compared to drugs). In addition, tDCS modifies spontaneous neuronal activity and therefore can increase activity in a more physiological manner and in addition be used while training is being performed [4]. Indeed several studies have shown that tDCS can increase cognitive gains when associated with behavioral interventions [4, 9-11].

The association of tDCS to the multidisciplinary intervention did not prove to be effective in reducing pain as measured by the VAS. However, the evaluation of pain according to the pain domain of SF-36 showed significant reduction only in the group that received active stimulation. These differences may result from some points that need to be discussed. First, the instrument of evaluation. VAS measurement of pain is related to the perception during the patient assessment. In this context, SF-36 is a more appropriate scale as it evaluates the previous 4-week period. Also, not only does this later instrument inquire about the amount of pain over the body in the last 4 weeks, but also to which extent it interferes with social life. Because the focus of multidisciplinary intervention was directed to behaviors, and not only pain perception, an instrument with broader range of observation, like social life participation, is more appropriated. This idea is supported by the trend toward a significant better improvement in quality of life according to the FIQ, which is also a multidimensional tool.

Another important aspect is that we performed weekly, rather than daily, sessions of tDCS and this strategy might be less effective according to a recent study in stroke subjects [30], although this later study was directed to the treatment of depression, not pain. The rationale for weekly sessions was based on the fact that tDCS has shown to induce cumulative effects based on the findings of such use in chronic pain [8] and depression [30]. Therefore we hypothesized that stimulation once a week might induce cumulative effects. However the number of consecutive sessions to induce cumulative effects might be important as these studies used daily tDCS on weekdays for 10 sessions and showed some persistence of favorable outcomes on a 2 
weeks follow-up. Stimulation of M1 was based firstly on the previous results of Fregni $[9,14]$, who proved it to be more effective than prefrontal stimulation. Also, it is hypothesized that the thalamus, and therefore rostrocaudal pain inhibitory neural pathways are stimulated by afferences from M1, resulting in reduction of pain perception [31].

Because improvement with active tDCS was observed only in one of the chosen outcomes, our results failed to fully support our hypothesis that weekly tDCS combined with a multidisciplinary approach is associated with beneficial effects for fibromyalgia. Therefore it is not clear whether this scenario with some significant findings and other lack of significant results is because our strategy of treatment (weekly rather than daily sessions), type of intervention (multidisciplinary approach) or instruments of evaluation. This question needs to be further explored in studies using other strategies of treatment. Alternatively, weekly anodal stimulation with tDCS over M1 may add no value over conventional rehabilitation for fibromyalgia.

Another critical issue for our mixed results is our small sample size that was based on positive results from a similar study, but that had a different context (daily tDCS sessions and no multidisciplinary treatment). Therefore it is possible that we were underpowered to detect a difference in the active $v s$ sham groups in the other domains. Moreover, because both groups were under rehabilitation treatment, a reduction of effect size between stimulated and sham groups should be expected, requiring a larger sample to evidence the difference. However, we also considered that a significant difference with a larger population would not show a clinically meaningful result and in addition, in some of the tests, there was not even a trend for a significant result. Moreover, patient population was different as compared to Fregni's study, in which patients did not undergo a simultaneous multidisciplinary approach $[9,14]$, and therefore might also explain the differences. Although it can be said that to many variables were involved in the intervention, like drugs, tDCS and multidisciplinary approach, it is important to remember that our object was to check the efficacy of the association of interventions, rather than each of them isolated. Because fibromyalgia is a chronic condition, a follow-up assessment would be desired, but this was not planned in this study. Another limitation of this study is the fact that multiple comparisons were performed without corrections, like Bonferrogni, so there in a possibility that the few positive finding are only due to chance. Finally the results of this study should be viewed as exploratory as to design further studies on this field.

\section{CONCLUSION}

This study could only partially show that tDCS may be an effective add-on treatment to a multidisciplinary approach in patients with fibromyalgia to reduce pain. Furthermore, a trend toward better global functioning was suggested regardless of tDCS. Future studies should test different protocols of stimulation with daily sessions of tDCS and also a maintenance treatment.

\section{ACKNOWLEDGEMENTS}

This study has been conducted with institutional resources, it is original and the authors have no personal monetary benefit with it.

\section{CONFLICT OF INTEREST}

\author{
None declared.
}

\section{REFERENCES}

[1] Wolfe F, Smythe HA, Yunus MB, et al. The American College of Rheumatology 1990 criteria for the classification of fibromyalgia. Report of the multicenter criteria committee. Arthitis Rheum 1990; 33: 160-72.

[2] Yunus MB. Fibromyalgia and overlapping disorders: the unifying concept of central sensitivity syndromes. Arhritis Rheum 2007; 36: 339-56.

[3] Burgmer M, Pogatzki-Zahn E, Gaubit M, Wessoleck E, Heuft G, Pfleiderer B. Altered brain activity during pain processing in fibromyalgia. Neuroimage 2009; 44: 502-8.

[4] Nitsche MA, Liebetanz D, Antal A, Lang N, Tergau F, Paulus W. Modulation of cortical instability by weak direct stimulation technical, safety and functional aspects. Suppl Clin Neurophysiol 2003; 56: 255-76.

[5] Nitsche MA, Fricke K, Henschke U, et al. Pharmacological modulation of cortical excitability shifts induced by transcranial direct current stimulation in humans. J Physiol 2003; 553, 293-301.

[6] Wagner T, Fregni F, Fecteau S, Grodzinsky A, Zahn M, PascualLeone A. Transcranial direct current stimulation: a computer-based human model study. Neuroimage 2007; 35(3): 1113-24.

[7] Boros K, Poreisz C, Münchau A, Paulus W, Nitsche MA. Premotor trancranial direct current stimulation (tDCS) affects primary motor excitability in humans. Eur J Neurosci 2008; 27(5): 1292-300.

[8] Fregni F, Boggio PS, Lima MC, et al. A sham-controlled, phase II trial of transcranial direct current stimulation for the treatment of central pain in traumatic spinal cord injury. Pain 2006; 122(1-2): 197-209.

[9] Fregni F, Gimenes R, Valle AC, et al. A randomized, shamcontrolled, proof-of-principle study of transcranial direct current stimulation for the treatment of pain in fibromyalgia. Arthritis Rheum 2006; 54(12): 3988-98.

[10] Hummel F, Cohen LG. Improvement of motor function with noninvasive cortical stimulation in a patient with chronic stroke. Neurorehabil Neural Repair 2005; 19(1): 14-9.

[11] Flöel A, Rösser N, Michka O, Knecht S, Breitenstein C. Noninvasive brain stimulation improves language learning. J Cogn Neurosci 2008; 81-8.

[12] Cruciani RA, Esteban S, Sibirceva U, Knotkova H. Non-invasive brain stimulation therapy for the management of complex regional pain syndrome (CRPS). J Pain Management 2009; 2: 277-84.

[13] Antal A, Paulus W, Nitsche MA. Principles and mechanisms of transcranial Direct Current Stimulation (tDCS). J Pain Management 2009; 2: 249-58.

[14] Valle A, Roizemblatt S, Botte S, et al. Efficacy of transcranial direct current stimulation (tDCS) for the treatment of fibromyalgia: results of a randomized, sham-controlled longitudinal clinical trial. J Pain Manag 2009; 2: 353-62.

[15] Fregni F, Boggio PS, Nitsche M, et al. Anodal transcranial direct current stimulation of prefrontal cortex enhances working memory. Exp Brain Res. 2005; 166(1): 23-30.

[16] Jorge LL, Tomikawa LCO, Jucá SSH. Effects of a rehabilitation program for men with fibromyalgia: a randomized controlled study. Acta Fisiatr 2007; 14(4): 196-203.

[17] Gandiga PC, Hummel F, Cohen LG. Transcranial DC stimulation (tDCS): a tool for double-blind sham-controlled clinical studies in brain stimulation. Clin Neurophysiol 2006; 117(4): 845-50.

[18] Gracely RH, Grant MA, Giesecke T. Evoked pain measures in fibromyalgia. Best Pract Res Rheumatol 2003; 17(4): 593-609.

[19] Martinez JE, Ferraz MB, Sato EI, Atra E. Fibromyalgia versus rheumatoid arthritis: a longitudinal comparison of quality of life. J Rheumatol 1995; 22(2): 270-4.

[20] Mota Falcão D, Ciconelli RM, Ferraz MB. Translation and cultural adaptation of quality of life questionnaires: an evaluation of methodology. J Rheumatol 2003; 30(2): 379-85.

[21] Bruce B, Fries JF The Stanford health assessment questionnaire (HAQ): a review of its history, issues, progress, and documentation. J Rheumatol 2003; 30: 167-178.

[22] Gorenstein C, Andrade L. Validation of a Portuguese version of the Beck Depression Inventory and State-Trait Anxiety Inventory in Brazilian subjects. Braz J Med Biol Res 1996; 29(4): 453-7. 
[23] Carville SF, Arendt-Nielsen S, Bliddal H, et al. EULAR evidence based recommendations for the management of fibromyalgia syndrome. Ann Rheum Dis 2008; 67: 536-41.

[24] Assis MR, Silva LE, Alves AM, et al. A randomized controlled trial of deep water running: clinical effectiveness of aquatic exercise to treat fibromyalgia. Arthritis Rheum 2006; 55(1): 57-65.

[25] Rooks DS, Gautam S, Romeling M, et al. Group exercise, education, and combination self-management in women with fibromyalgia: a randomized trial. Arch Intern Med 2007; 167(20): 2192-200.

[26] Arnold LM. Duloxetine and other antidepressant in the treatment of patients with fibromyalgia. Pain Med; 8(suppl2): s63-74.

[27] Furlan AD, Sandoval JA, Mailis-Ganon A, Tunk E. Opioids for noncancer pain: a meta-analysis of effectiveness and side effects. CMAJ 2006; 174(11): 1589-94
[28] Dunkl PR, Taylor AG, McConnell GG, Alfano AP, Conaway MR. Responsiveness of fibromialgia clinical trial outcomes measures. Rheumatol 2000; 27: 2683-91.

[29] Short B, Borckardt JJ, George M, Beam W, Reeves ST. Noninvasive brain stimulation approaches to fibromyalgia pain. J Pain management 2009; 2: 259-76.

[30] Boggio PS, Rigonatti SP, Ribeiro RB, et al. A randomized, doubleblind clinical trial on the efficacy of cortical direct current stimulation for the treatment of major depression. Int Neuropsychopharmacol 2007; 11: 1-6.

[31] Lima MC, Fregni F. Motor cortex stimulation for chronic pain: systematic review and meta-analysis of the literature. Neurology. 2008; 70(24): 2329-37.

(C) Riberto et al.; Licensee Bentham Open.

This is an open access article licensed under the terms of the Creative Commons Attribution Non-Commercial License (http://creativecommons.org/licenses/by-nc/ 3.0/) which permits unrestricted, non-commercial use, distribution and reproduction in any medium, provided the work is properly cited 